\title{
BMJ Open Medical claims-based case-control study of temporal relationship between clinical visits for hand syndromes and subsequent diabetes diagnosis: implications for identifying patients with undiagnosed type 2 diabetes mellitus
}

\author{
Wen-Hsuan Hou, ${ }^{1,2,3,4}$ Chung-Yi Li, ${ }^{5,6}$ Lu-Hsuan Chen, ${ }^{5,6}$ Liang-Yi Wang, ${ }^{5}$ \\ Li-Chieh Kuo, ${ }^{7}$ Ken N Kuo, ${ }^{1}$ Hsiu-Nien Shen, ${ }^{8}$ Chang-Ta Chiu ${ }^{9}$
}

To cite: Hou W-H, Li C-Y, Chen L-H, et al. Medical claims-based case-control study of temporal relationship between clinical visits for hand syndromes and subsequent diabetes diagnosis: implications for identifying patients with undiagnosed type 2 diabetes mellitus. BMJ Open 2016;6: e012071. doi:10.1136/ bmjopen-2016-012071

- Prepublication history for this paper is available online. To view these files please visit the journal online (http://dx.doi.org/10.1136/ bmjopen-2016-012071).

H-NS and C-TC contributed equally.

Received 28 March 2016 Revised 3 October 2016 Accepted 4 October 2016

CrossMark

For numbered affiliations see end of article.

Correspondence to Dr Chang-Ta Chiu; chiouchangta@yahoo.com.tw

\section{ABSTRACT}

Objectives: To investigate whether a temporal relationship is present between clinical visits for diabetes-related hand syndromes (DHSs) and subsequent type 2 diabetes mellitus (T2DM) diagnosis and, accordingly, whether DHSs can be used for identifying patients with undiagnosed T2DM.

Design: This study had a case-control design nested within a cohort of 1 million people from the general population, which was followed from 2005 to 2010 . The odds of prior clinical visits for DHSs, namely carpal tunnel syndrome (CTS), flexor tenosynovitis, limited joint mobility and Dupuytren's disease, were estimated for cases and controls. We used a conditional logistic regression model to estimate the $\mathrm{OR}$ and $95 \% \mathrm{Cl}$ of T2DM in association with a history of DHSs. The validity and predictive value of using the history of DHSs in predicting T2DM diagnosis were calculated.

Setting: Taiwan National Health Insurance medical claims.

Participants: We identified 33571 patients receiving a new diagnosis of T2DM (cases) between 2005 and 2010. Each T2DM case was matched with 5 controls who had the same sex and birth year and were alive on the date of T2DM diagnosis.

Primary and secondary outcome measures: The primary outcome measure was T2DM diagnosis.

Results: The OR of T2DM in association with prior clinical visits was significantly increased for overall DHS and CTS, being 1.15 (95\% Cl 1.10 to 1.20 ) and 1.22 (95\% Cl 1.16 to 1.29$)$, respectively. Moreover, $11 \%$ of patients with T2DM made clinical visits for CTS within 3 months prior to T2DM diagnosis. The history of DHSs had low sensitivity $(<0.1 \%$ to $5.2 \%)$ and a positive predictive value $(9.9 \%$ to $11.7 \%)$ in predicting T2DM. Conclusions: Despite the unsatisfactory validity and performance of DHSs as a clinical tool for detecting patients with undiagnosed T2DM, this study provided

\section{Strengths and limitations of this study}

- The nested case-control design used in this study has a low likelihood of selection bias.

- The sample size of this study is sufficiently large to yield adequate statistical power.

- Potential disease misclassification resulting from the use of medical claims tends to underestimate rather than overestimate the temporal relationship between clinical visits for diabetes-related hand syndromes and subsequent type 2 diabetes mellitus diagnosis.

- The study findings should be interpreted with caution because not all known risk factors for type 2 diabetes mellitus were considered in the analysis.

evidence that clinical visits for DHSs, particularly for CTS, can be a sign of undiagnosed T2DM.

\section{INTRODUCTION}

Diabetes is one of the most common metabolic disorders worldwide, and its prevalence in adults has increased in past decades. ${ }^{1}$ The International Diabetes Federation estimated that 381.8 million people had diabetes in 2013 and predicted that this number will increase to 591.9 million (projected increase of $55 \%$ ) by $2035 .^{2}$ In addition, the prolonged asymptomatic phase of type 2 diabetes mellitus (T2DM) may last for many years. ${ }^{3}$ The non-management of increased blood glucose levels during this phase can lead to severe 
complications including neuropathy, nephropathy, retinopathy, coronary artery disease, stroke or peripheral vascular disease, ${ }^{4}$ resulting in massive healthcare costs and a global health burden. ${ }^{5}$

Studies have indicated that a high proportion of undiagnosed T2DM-related and diabetes-related complications may be attributable to underperforming health systems, low awareness among the general public and health professionals, and slow symptom onset or progression of T2DM; hyperglycaemic conditions may remain undetected for many years. ${ }^{6} 7$ Although blood sugar screening has been considered the most effective method for early and timely diagnosis of T2DM, inadequate healthcare services, poor health literacy and lack of active health behaviour still impede the success of blood sugar screening campaigns, particularly in rural areas and in those who are poor. ${ }^{8}$ Therefore, the inspection of possible diabetes-related syndromes in clinical settings should be considered as an additional method for the early identification of undiagnosed T2DM.

Diabetes-related hand syndromes (DHSs), defined as certain musculoskeletal conditions of the hands, constitute a clinical problem in patients with diabetes and are almost invariably associated with long-standing diabetes, suboptimal glycaemic control and microvascular complications. $^{9-13}$ DHSs include limited joint mobility (LJM) ${ }^{12}{ }^{14-16}$ stenosing flexor tenosynovitis (SFT), ${ }^{11} 1718$ Dupuytren's disease (DD) ${ }^{17} 1920$ and carpal tunnel syndrome (CTS). ${ }^{15} 172122$ A study suggested that clinicians should emphasise the clinical examination of DHSs and use DHSs as a clinical diagnostic tool for T2DM because of the significant association of the duration of T2DM with the prevalence of DHSs. ${ }^{23}$ However, DHSs have received less attention compared with other diabetic complications, such as diabetic foot problems and cardiovascular disease, which both patients with diabetes and healthcare professionals are familiar with. Therefore, this study investigated whether the risk of T2DM increases after clinical visits for DHSs. In other words, we examined whether DHSs can be considered an indicator of undiagnosed T2DM.

\section{METHODS}

\section{Data sources}

Data analysed in this study were retrieved from the medical claims of the National Health Insurance Research Database (NHIRD) provided by the National Health Insurance Administration (NHIA), Ministry of Health and Welfare, Taiwan. Approximately $92.3 \%$ of the residents of Taiwan were enrolled in the National Health Insurance (NHI) programme by the end of 1996, and the coverage has increased to $99 \%$ since 2004. ${ }^{24} 25$ The NHIRD contains all inpatient and outpatient claims data and medical records and information on healthcare providers, including medical institutions and healthcare workers. The personal identification numbers of all beneficiaries are encrypted to ensure privacy. To ensure the accuracy of claim files, the NHIA performs quarterly expert reviews on a random sample for every 50-100 ambulatory and inpatient claims. ${ }^{26}$ Access to research data has been reviewed and approved by the Review Committee of the National Health Research Institutes (NHRI).

\section{Nested case-control design}

This study was based on the claims data from 1997 to 2010 of one million beneficiaries randomly selected from all beneficiaries registered in 2005. We excluded patients who were aged $<20$ years $(n=394377)$ on the first day of 2005 and had a history of type 1 diabetes mellitus $(n=2507)$ and T2DM ( $\mathrm{n}=61$ 966) from 1997 to 2004. Finally, we included a total of 541150 patients in the study cohort. By the end of 2010, we identified 33571 incident cases of T2DM (International Classification of Diseases, Ninth Revision, Clinical Modification (ICD-9-CM) code 250.xx excluding 250.x1 and 250.x3).

For each T2DM case, we randomly selected five controls by using the incidence density sampling technique. ${ }^{27}$ Each T2DM case was matched with five controls who had the same sex and birth year and were alive on the date of T2DM diagnosis. Therefore, people could be selected as controls before they received the diagnosis of T2DM. In addition, a person could serve as a control for multiple cases. The density sampling finally resulted in a total of 167777 controls.

\section{Outcome measures}

The outcome variable was the diagnosis of T2DM. We included only those patients who received a diagnosis of T2DM between 2005 and 2010 and again within the subsequent 12 months; the first and last ambulatory care visits (including both hospital outpatients and general practice) for T2DM during the 12-month period had to be separated by at least 30 days. This prevented accidental inclusion of miscoded patients. ${ }^{28} 29$ In addition, the first and last outpatient visits during the 12-month period had to be separated by at least 30 days to prevent accidental inclusion of miscoded patients. ${ }^{30}$

\section{Prior history of DHSs}

Four DHSs were included as primary independent variables, namely CTS (ICD-9-CM code 354.0), SFT (ICD-9-CM code 727.03), LJM (ICD-9-CM code 718.8) and DD (ICD-9-CM code 728.6). Information on the history of DHSs was retrieved from ambulatory care visits (including hospital outpatients and general practice) made between 1 January 1997 and the date of diagnosis of incident T2DM. In Taiwan, physicians from various specialties can diagnose DHSs.

\section{Statistical analysis}

We first examined the distribution of covariates between cases and controls. We then analysed the data by using conditional logistic regression models and estimated 
ORs and their $95 \%$ CIs of prior ambulatory care visits for DHSs in association with T2DM. Crude ORs were estimated using simple conditional logistic regression that accounted for matching variables such as age, sex and the date of incident T2DM diagnosis in the analysis. Moreover, we adjusted for the area of residence (north, central, south and east), occupation (blue collar, white collar and unclassified) and monthly income based on the insurance premium in the conditional logistic regression model.

We considered the insurance premium and occupation as covariates mainly because studies have reported that low socioeconomic status and exposure to some work hazards may increase the risk of the selected DHSs. ${ }^{31-33}$ Moreover, adjustment for the geographic area helped in reducing the presence of a geographic difference in accessibility to medical health services in Taiwan. ${ }^{34}$

To assess the validity of using the selected hand syndromes in identifying the cases of T2DM, we calculated the sensitivity and specificity of each selected hand syndrome. With respect to the performance of using the history of DHSs in predicting the incidence of T2DM, we estimated the positive predictive value (PPV) and negative predictive value (NPV) on the basis of the Bayesian approach. ${ }^{35}$ To estimate the PPV and NPV, the prevalence of T2DM was set at $9.9 \%$ according to a recent Taiwanese survey. ${ }^{36}$
The statistical analyses were performed using SAS (V.9.4; SAS Institute, Cary, North Carolina, USA), and a $p$ value of $<0.05$ was considered statistically significant.

\section{RESULTS}

The area of residence, occupation and salary-based insurance premium differed significantly between the cases and controls. The case patients were less likely to live in the central region, were more likely to be bluecollar workers and paid lower insurance premiums for dependants (table 1). Compared with the control people, the case patient had a significantly higher adjusted OR (1.31; 95\% CI 1.22 to 1.40$)$ of having a prior diagnosis of CTS and any type of DHS (adjusted OR, $1.23 ; 95 \%$ CI 1.16 to 1.30). Adjusted ORs associated with hand syndromes other than CTS were not significant (table 2).

Table 3 lists the time elapsed from the last ambulatory care visit for DHSs to the diagnosis of T2DM. In total, $11 \%$ of the case patients and only $5.8 \%$ of the controls had their last clinical visits for CTS 3 months prior to the diagnosis of T2DM. The proportions of case patients and controls who had their last clinical visits for CTS between 3 and 6 months, between 6 and 12 months, between 12 and 24 months, and after 24 months were similar. Such a pattern was not observed for other selected DHSs. Table 4 illustrates that the selected DHSs

\begin{tabular}{|c|c|c|c|}
\hline & $\begin{array}{l}\text { Cases } \\
\text { n (\%) }\end{array}$ & $\begin{array}{l}\text { Controls } \\
\text { n (\%) }\end{array}$ & p Value \\
\hline Age (years) & & & 0.9871 \\
\hline $20-34$ & 1007 (3.00) & 5015 (2.99) & \\
\hline $35-64$ & 22657 (67.49) & 113301 (67.53) & \\
\hline$\geq 65$ & 9907 (29.51) & $49461(29.48)$ & \\
\hline Sex & & & 0.9847 \\
\hline Men & 17904 (53.33) & 89488 (53.34) & \\
\hline Women & 15667 (46.67) & 78289 (46.66) & \\
\hline Area of residence & & & 0.0152 \\
\hline North & $15472(46.23)$ & 77085 (46.12) & \\
\hline Central & 7785 (23.26) & $39630(23.71)$ & \\
\hline South & 9178 (27.42) & 45752 (27.37) & \\
\hline East & $1034(3.09)$ & $4687(2.80)$ & \\
\hline Occupation & & & $<0.0001$ \\
\hline White collar & 7454 (30.74) & 41809 (33.60) & \\
\hline Blue collar & $11261(46.44)$ & 55724 (44.78) & \\
\hline Unclassified & $5533(22.82)$ & 26909 (21.62) & \\
\hline Insurance premium (NTD) & & & $<0.0001$ \\
\hline 0 (dependants) & $9221(27.55)$ & $42716(25.55)$ & \\
\hline $1-17280$ & $6363(19.01)$ & 31717 (18.97) & \\
\hline $17281-21000$ & $8610(25.73)$ & $43714(26.15)$ & \\
\hline $21001-34800$ & $4140(12.37)$ & 20069 (12.01) & \\
\hline$>34800$ & $5135(15.34)$ & $28942(17.31)$ & \\
\hline Total $^{*}$ & 33571 & 167777 & \\
\hline
\end{tabular}


Table 2 Crude and adjusted OR of type 2 diabetes mellitus in association with a history of diabetes-related hand syndromes

\begin{tabular}{|c|c|c|c|c|c|c|c|c|}
\hline & \multicolumn{2}{|l|}{ Controls } & \multicolumn{2}{|l|}{ Cases } & \multicolumn{2}{|c|}{ Crude estimates } & \multicolumn{2}{|c|}{ Adjusted estimates } \\
\hline & $\mathrm{n}$ & Per cent & $\mathrm{n}$ & Per cent & OR & $95 \% \mathrm{Cl}$ & OR & $95 \% \mathrm{Cl}$ \\
\hline \multicolumn{9}{|l|}{ CTS } \\
\hline Yes & 7271 & 4.3 & 1754 & 5.2 & 1.22 & 1.16 to 1.29 & 1.31 & 1.22 to 1.40 \\
\hline No & 160506 & 95.7 & 31817 & 94.8 & Ref. & & Ref. & \\
\hline \multicolumn{9}{|l|}{ SFT } \\
\hline Yes & 4061 & 2.4 & 816 & 2.4 & 1.01 & 0.93 to 1.09 & 1.05 & 0.95 to 1.16 \\
\hline No & 163716 & 97.6 & 32755 & 97.6 & Ref. & & Ref. & \\
\hline \multicolumn{9}{|l|}{ LJM } \\
\hline Yes & 337 & 0.2 & 69 & 0.2 & 1.02 & 0.79 to 1.33 & 1.05 & 0.75 to 1.47 \\
\hline No & 167440 & 99.8 & 32502 & 99.8 & Ref. & & Ref. & \\
\hline \multicolumn{9}{|l|}{ DD } \\
\hline Yes & 27 & 0.02 & 6 & 0.02 & 1.11 & 0.46 to 2.69 & 1.13 & 0.42 to 3.03 \\
\hline No & 167750 & 99.98 & 33565 & 99.98 & Ref. & & Ref. & \\
\hline \multicolumn{9}{|c|}{ Any of the above } \\
\hline Yes & 11610 & 6.9 & 2629 & 7.8 & 1.15 & 1.10 to 1.20 & 1.23 & 1.16 to 1.30 \\
\hline No & 156167 & 93.1 & 30942 & 92.2 & Ref. & & Ref. & \\
\hline Total & 167777 & & 33571 & & & & & \\
\hline
\end{tabular}

Table 3 Distribution of the time elapsed from the date of the last diagnosis of diabetes-related hand syndromes to the date of the diagnosis of type 2 diabetes mellitus between cases and controls

\begin{tabular}{|c|c|c|c|c|c|c|c|c|c|c|c|}
\hline & \multicolumn{10}{|c|}{ Time elapsed (months) } & \multirow[b]{3}{*}{ p Value* } \\
\hline & \multicolumn{2}{|l|}{$<3$} & \multicolumn{2}{|c|}{3 to $<6$} & \multicolumn{2}{|c|}{6 to $<12$} & \multicolumn{2}{|c|}{12 to $<24$} & \multicolumn{2}{|l|}{$\geq 24$} & \\
\hline & $\bar{n}$ & Per cent & $\bar{n}$ & Per cent & $\overline{\mathbf{n}}$ & Per cent & $\bar{n}$ & Per cent & $\bar{n}$ & Per cent & \\
\hline \multicolumn{12}{|l|}{ CTS } \\
\hline Cases & 193 & 11.0 & 74 & 4.2 & 138 & 7.9 & 235 & 13.4 & 1114 & 63.5 & $<0.001$ \\
\hline Controls & 422 & 5.8 & 288 & 4.0 & 540 & 7.4 & 949 & 13.1 & 5072 & 69.8 & \\
\hline \multicolumn{12}{|l|}{ SFT } \\
\hline Cases & 52 & 6.4 & 39 & 4.8 & 90 & 11.0 & 129 & 15.8 & 506 & 62.0 & 0.216 \\
\hline Controls & 284 & 7.0 & 226 & 5.6 & 359 & 8.8 & 593 & 14.6 & 2599 & 64.0 & \\
\hline \multicolumn{12}{|l|}{ LJM } \\
\hline Cases & 4 & 5.8 & 2 & 2.9 & 5 & 7.3 & 9 & 13.0 & 49 & 71.0 & 0.413 \\
\hline Controls & 11 & 3.3 & 8 & 2.4 & 22 & 6.5 & 27 & 8.0 & 269 & 80.0 & \\
\hline \multicolumn{12}{|l|}{ DD } \\
\hline Cases & 0 & 0.0 & 0 & 0.0 & 0 & 0.0 & 1 & 16.7 & 5 & 83.3 & 0.821 \\
\hline Controls & 2 & 7.4 & 0 & 0.0 & 3 & 11.1 & 5 & 18.5 & 17 & 63.0 & \\
\hline
\end{tabular}

*Based on Pearson's $\chi^{2}$ test or Fisher's exact test.

CTS, carpal tunnel syndrome; DD, Dupuytren's disease; LJM, limited joint mobility; SFT, stenosing flexor tenosynovitis.

had very low sensitivity in identifying patients with T2DM. The highest sensitivity was noted for CTS $(5.2 \%)$. In addition, the PPV for the selected syndromes was low, ranging from $9.9 \%$ for DD to $11.7 \%$ for CTS.

\section{DISCUSSION}

The results of this population-based nested case-control study indicated that compared with the controls, the patients with T2DM had a significantly higher likelihood of a history of CTS and any type of DHS (adjusted $\mathrm{OR}=1.31$ and 1.23 , respectively). Regarding the validity and performance of DHSs as a clinical tool for detecting patients with undiagnosed T2DM, both the sensitivity (5.2\% for CTS was the highest) and PPV (9.9\% for DD and $11.7 \%$ for CTS) were not satisfactory. Nevertheless, to the best of our knowledge, this is the first study to examine the temporal relationship between DHSs and T2DM and to investigate whether DHSs can be considered an indicator of undiagnosed T2DM in clinical settings.

Although a significant temporal relationship was present between overall DHS and the subsequent risk of T2DM, only CTS had a significant association with T2DM in the analyses of specific DHSs. Perkins $e t a l^{37}$ reported that the prevalence of clinical CTS was $14 \%$ in patients with diabetes and without diabetic peripheral neuropathy (DPN), 30\% in those with DPN and $2 \%$ in the reference population. Furthermore, they indicated that CTS, which is an entrapment neuropathy, is 
Table 4 Validity and performance of using diabetesrelated hand syndromes as a clinical tool for identifying people with undiagnosed type 2 diabetes mellitus

\begin{tabular}{lrrrr}
\hline & CTS & SFT & LJM & \multicolumn{1}{c}{ DD } \\
\hline Sensitivity (\%) & 5.2 & 2.4 & 0.2 & $<0.1$ \\
Specificity (\%) & 95.7 & 97.6 & 99.8 & 99.9 \\
PPV (\%) & 11.7 & 9.9 & 10.3 & 9.9 \\
NPV (\%) & 90.2 & 90.1 & 90.1 & 90.1 \\
\hline
\end{tabular}

CTS, carpal tunnel syndrome; DD, Dupuytren's disease; LJM, limited joint mobility; NPV, negative predictive value; PPV, positive predictive value; SFT, stenosing flexor tenosynovitis.

prevalent in patients with distal sensory peripheral neuropathy. In addition, Bahrmann $e t a l^{38}$ reported that the prevalence of CTS was higher in patients with diabetes and peripheral neuropathy than in patients with diabetes and without diabetes-related late complications (30\% vs $14 \%$ ). Moreover, CTS appears to be a risk factor for late manifestation of diabetes because patients receiving a new diagnosis of diabetes exhibited CTS manifestation 1.4-fold more often than did an agematched reference population. This relative risk estimate is consistent with that in our study (ie, adjusted OR 1.31; $95 \%$ CI 1.22 to 1.40 ). Our study results revealed that T2DM was associated with prior CTS, but not with other DHSs, suggesting that CTS is associated with peripheral neuropathy, which is a definite feature of pre-diabetes, ${ }^{39}$ whereas other DHSs are of musculoskeletal origin.

The temporal sequence of overall DHS and the subsequent diagnosis of T2DM observed in our study indicate that some patients who seek medical care for DHSs may have undiagnosed T2DM. The higher risk of DHSs in patients with T2DM may be attributable to several mechanisms. The accumulation of advanced glycosylation end products and glycated proteins or lipids after exposure to sugars may lead to abnormal cross-linking of collagen fibres, which becomes manifest as skin thickening and nodule and contracture formation on the hands. ${ }^{40}$ Other studies have supported the strong association of the increased risk of DHSs after T2DM diagnosis with the abnormal expression of some peptides and subclinical activation of specific cytokines, such as transforming growth factor- $\beta$, basic fibroblast growth factor, interleukin 1 (IL-1), IL-6 and tumour necrosis factor- $\alpha$, which may also lead to unregulated and abnormal proliferation of collagen. ${ }^{41} 42$

In Taiwan, numerous patients seek medical services directly from specialists without initially visiting general practitioners. Since DHSs involve the nerves or soft tissues of the hands, patients with such hand syndromes usually visit orthopaedists, neurologists or physiatrists. According to our data, more than two-thirds of the patients with DHSs received the diagnosis from orthopaedists, neurologists or physiatrists who did not have adequate experience in treating patients with T2DM in their clinical settings. Our data tended to exhibit an underdiagnosis of T2DM in some patients with DHSs, implying that these specialists might not be aware of the association of DHSs with T2DM. Although a standard definition is available for the diagnosis of DHSs, which is mainly dependent on ultrasonographic and functional assessment, ${ }^{43} 44$ the diagnosis of DHSs in ambulatory care settings could be subject to error because of limited diagnostic resources in some clinics. In addition, although patients with diabetes and healthcare professionals have high awareness regarding some diabetic complications other than DHSs, such as diabetic foot problems and cardiovascular disease, no studies have investigated the proportion of T2DM diagnosed according to the clinical appearance of these common diabetic complications. The ability of diabetes-related foot problems and cardiovascular disease to identify undiagnosed T2DM should be explored in future studies.

This study has the following strengths. First, it was a population-based study including a highly representative sample of patients with T2DM in Taiwan between 2005 and 2010. The results can be applied and generalised to patients with varying diabetes severities or to those from different clinical settings. Second, the advantage of using insurance claim data in clinical research is that it provides easy access to longitudinal records for a large sample of demographically diverse patients. ${ }^{45}{ }^{46}$ The size of the data set enabled stratified analyses to be conducted according to different time intervals between the diagnosis of DHSs and development of T2DM. Third, the T2DM cases and controls in this nested case-control study were collected from the NHI database, and all the research information was retrieved from the NHI claims, which minimised the likelihood of non-response or loss to follow-up of the study patients. Our study has some limitations. First, exclusive reliance on claims data may have resulted in a disease misclassification bias. The number of DHSs estimated from the claims data could be biased because some people who experienced DHS-related symptoms may have not sought ambulatory care, which would in turn lead to the underestimation of relative risk estimates. Second, our study might be subject to surveillance bias because patients who frequently visited clinics for DHSs may have an increased risk of T2DM later. Nevertheless, the risk of T2DM was significantly associated with CTS, but not with the other three DHSs, suggesting that the association of T2DM with DHSs or particularly with CTS may not entirely be explained by the potential surveillance bias. Third, the hand complications are more likely to be found in people who have had diabetes for a number of years, which limits their usefulness as indicators of pre-diabetes or early-stage diabetes.

\section{CONCLUSION}

Our study results revealed that clinical visits for DHSs can be a sign of undiagnosed T2DM. Despite the unsatisfactory validity and performance of DHSs as a clinical tool for detecting patients with undiagnosed T2DM, the 
awareness regarding the association of DHSs with undiagnosed T2DM is crucial to both the public and clinicians, particularly to non-internists.

\section{Author affiliations}

${ }^{1}$ Cochrane Taiwan, Taipei Medical University, Taipei, Taiwan

${ }^{2}$ Master Program in Long-Term Care, College of Nursing, Taipei Medical University, Taipei, Taiwan

${ }^{3}$ School of Gerontology Health Management, College of Nursing, Taipei Medical University, Taipei, Taiwan

${ }^{4}$ Department of Physical Medicine and Rehabilitation, Taipei Medical University Hospital, Taipei, Taiwan

${ }^{5}$ Department and Graduate Institute of Public Health, College of Medicine, National Cheng Kung University, Tainan, Taiwan

${ }^{6}$ Department of Public Health, College of Public Health, China Medical University, Taichung, Taiwan

${ }^{7}$ Department of Occupational Therapy, College of Medicine, National Cheng Kung University, Tainan, Taiwan

${ }^{8}$ Department of Intensive Care Medicine, Chi Mei Medical Center, Tainan, Taiwan

${ }^{9}$ Department of Dentistry, Tainan Municipal An-Nan Hospital, China Medical University, Tainan, Taiwan

Contributors $\mathrm{W}-\mathrm{HH}, \mathrm{L}-\mathrm{HC}$ and $\mathrm{C}-\mathrm{YL}$ designed the study. $\mathrm{L}-\mathrm{HC}$ and $\mathrm{L}-\mathrm{YW}$ analysed the data. W-HH, L-YW, L-CK, H-NS and C-TC drafted the manuscript. L-CK, KNK, H-NS, C-YL and C-TC reviewed and revised the manuscript.

Funding This study was supported by grants from the Ministry of Science and Technology (MOST; grant number 104-2314-B-006-020-MY2) and Chi Mei Medical Center (grant number 105-CM-TMU-02).

Disclaimer The interpretation and conclusions contained here do not represent those of the National Health Insurance Administration (NHIA) and National Health Research Institutes (NHRI).

Competing interests None declared.

Ethics approval The Institutional Review Board of the National Cheng Kung University.

Provenance and peer review Not commissioned; externally peer reviewed.

Data sharing statement No additional data are available.

Open Access This is an Open Access article distributed in accordance with the Creative Commons Attribution Non Commercial (CC BY-NC 4.0) license, which permits others to distribute, remix, adapt, build upon this work noncommercially, and license their derivative works on different terms, provided the original work is properly cited and the use is non-commercial. See: http:// creativecommons.org/licenses/by-nc/4.0/

\section{REFERENCES}

1. Shaw JE, Sicree RA, Zimmet PZ. Global estimates of the prevalence of diabetes for 2010 and 2030. Diabetes Res Clin Pract 2010;87:4-14.

2. Guariguata L, Whiting DR, Hambleton I, et al. Global estimates of diabetes prevalence for 2013 and projections for 2035. Diabetes Res Clin Pract 2014;103:137-49.

3. American Diabetes A. Diagnosis and classification of diabetes mellitus. Diabetes Care 2012;35(Suppl 1):S64-71.

4. Malandrino N, Wu WC, Taveira TH, et al. Association between red blood cell distribution width and macrovascular and microvascular complications in diabetes. Diabetologia 2012;55:226-35.

5. O'Brien JA, Patrick AR, Caro J. Estimates of direct medical costs for microvascular and macrovascular complications resulting from type 2 diabetes mellitus in the United States in 2000. Clin Ther 2003;25:1017-38

6. Beagley J, Guariguata L, Weil C, et al. Global estimates of undiagnosed diabetes in adults. Diabetes Res Clin Pract 2014;103:150-60.

7. Herman $\mathrm{WH}$, Kennedy L. Underdiagnosis of peripheral neuropathy in type 2 diabetes. Diabetes Care 2005;28:1480-1.
8. King H, Rewers M. Global estimates for prevalence of diabetes mellitus and impaired glucose tolerance in adults. WHO Ad Hoc Diabetes Reporting Group. Diabetes Care 1993;16:157-77.

9. Ceruso M, Lauri G, Bufalini C, et al. Diabetic hand syndrome. $J$ Hand Surg Am 1988;13:765-70.

10. Rosenbloom AL. Limitation of finger joint mobility in diabetes mellitus. J Diabet Complications 1989;3:77-87.

11. Blyth MJ, Ross DJ. Diabetes and trigger finger. $J$ Hand Surg $\mathrm{Br}$ 1996;21:244-5.

12. Lekholm C, Sundkvist G, Lundborg G, et al. [The diabetic handcomplications of diabetes]. Lakartidningen 2001;98:306-12.

13. Thornton DJ, Lindau T. Hand infections. Orthop Trauma 2010;24:186-96.

14. Papanas N, Maltezos E. The diabetic hand: a forgotten complication? J Diabet Complications 2010;24:154-62.

15. Gamstedt A, Holm-Glad J, Ohlson CG, et al. Hand abnormalities are strongly associated with the duration of diabetes mellitus. J Intern Med 1993;234:189-93.

16. Aljahlan $\mathrm{M}$, Lee $\mathrm{KC}$, Toth $\mathrm{E}$. Limited joint mobility in diabetes. Postgrad Med 1999;105:99-101, 105-6.

17. Chammas $M$, Bousquet $P$, Renard $E$, et al. Dupuytren's disease, carpal tunnel syndrome, trigger finger, and diabetes mellitus. J Hand Surg Am 1995;20:109-14.

18. Stahl S, Kanter Y, Karnielli E. Outcome of trigger finger treatment in diabetes. J Diabetes Complicat 1997;11:287-90.

19. Jennings AM, Milner PC, Ward JD. Hand abnormalities are associated with the complications of diabetes in type 2 diabetes. Diabet Med 1989;6:43-7.

20. Arkkila PE, Kantola IM, Viikari JS. Dupuytren's disease: association with chronic diabetic complications. J Rheumatol 1997;24:153-9.

21. Stamboulis E, Voumvourakis K, Andrikopoulou A, et al. Association between asymptomatic median mononeuropathy and diabetic polyneuropathy severity in patients with diabetes mellitus. J Neurol Sci 2009:278:41-3.

22. Karpitskaya Y, Novak CB, Mackinnon SE. Prevalence of smoking, obesity, diabetes mellitus, and thyroid disease in patients with carpa tunnel syndrome. Ann Plast Surg 2002;48:269-73.

23. Al-Matubsi HY, Hamdan F, Alhanbali OA, et al. Diabetic hand syndromes as a clinical and diagnostic tool for diabetes mellitus patients. Diabetes Res Clin Pract 2011;94:225-9.

24. Lu JF, Hsiao WC. Does universal health insurance make health care unaffordable? Lessons from Taiwan. Health Aff (Millwood) 2003;22:77-88.

25. Ministry of Health and Welfare. Main indicators of statistics and surveys. 2015. http://www.nhi.gov.tw/English/webdata/webdata. aspx?menu=11\&menu_id=296\&webdata_id=1942\&WD_ID=296 (accessed 2 Aug 2016)

26. Insurance BoNH. Regulations governing contracting and management of National Health Insurance medical care institutions. 2014. http://www.nhi.gov.tw/English/webdata/webdata.aspx? menu=11\&menu_id=295\&WD_ID=295\&webdata_id $=2440$ (accessed 25 Jul 2015)

27. Greenland S, Thomas DC. On the need for the rare disease assumption in case-control studies. Am J Epidemiol 1982;116:547-53.

28. Chen HF, Ho CA, Li CY. Age and sex may significantly interact with diabetes on the risks of lower extremity amputation and peripheral revascularization procedures: evidence from a cohort of a half million diabetic patients. Diabetes Care 2006;29:2409-14.

29. Chen HF, Ho CA, Li CY. Increased risks of hip fracture in diabetic patients of Taiwan: a population-based study. Diabetes Care 2008;31:75-80.

30. Chen HF, Chen P, Li CY. Risk of malignant neoplasms of liver and biliary tract in diabetic patients with different age and sex stratifications. Hepatology 2010;52:155-63.

31. Burt S, Deddens JA, Crombie K, et al. A prospective study of carpal tunnel syndrome: workplace and individual risk factors. Occup Environ Med 2013;70:568-74.

32. Roquelaure $\mathrm{Y}, \mathrm{Ha} \mathrm{C}$, Rouillon $\mathrm{C}$, et al. Risk factors for upper-extremity musculoskeletal disorders in the working population. Arthritis Rheum 2009;61:1425-34.

33. Shiri R. Personal and workplace risk factors for carpal tunne syndrome. Occup Environ Med 2014;71:303.

34. Tan HF, Tseng HF, Chang CK, et al. Accessibility assessment of the Health Care Improvement Program in rural Taiwan. J Rural Health 2005;21:372-7.

35. Gordis L. More on causal inferences: bias, confounding, and interaction. In: Epidemiology. 2nd edn. Philadelphia: W.B. Saunders, 2000:204-21.

36. Jiang YD, Chang $\mathrm{CH}$, Tai TY, et al. Incidence and prevalence rates of diabetes mellitus in Taiwan: analysis of the 2000-2009 
Nationwide Health Insurance database. J Formos Med Assoc 2012;111:599-604.

37. Perkins BA, Olaleye D, Bril V. Carpal tunnel syndrome in patients with diabetic polyneuropathy. Diabetes Care 2002;25:565-9.

38. Bahrmann A, Zieschang T, Neumann T, et al. Carpal tunnel syndrome in diabetes mellitus. Med Klin (Munich) 2010;105:150-4.

39. Papanas N, Vinik Al, Ziegler D. Neuropathy in prediabetes: does the clock start ticking early? Nat Rev Endocrinol 2011;7:682-90.

40. Ravindran Rajendran S, Bhansali A, Walia R, et al. Prevalence and pattern of hand soft-tissue changes in type 2 diabetes mellitus. Diabetes Metab 2011;37:312-17.

41. Spranger J, Kroke A, Mohlig M, et al. Inflammatory cytokines and the risk to develop type 2 diabetes: results of the prospective population-based European Prospective Investigation into Cancer and Nutrition (EPIC)-Potsdam Study. Diabetes 2003;52:812-17.

42. Miyauchi K, Takiyama Y, Honjyo J, et al. Upregulated IL-18 expression in type 2 diabetic subjects with nephropathy: TGF-beta1 enhanced IL-18 expression in human renal proximal tubular epithelial cells. Diabetes Res Clin Pract 2009;83:190-9.

43. Chen SF, Huang CR, Tsai NW, et al. Ultrasonographic assessment of carpal tunnel syndrome of mild and moderate severity in diabetic patients by using an 8-point measurement of median nerve cross-sectional areas. BMC Med Imaging 2012;12:15.

44. Hsu HY, Kuo YL, Jou IM, et al. Diagnosis from functional perspectives: usefulness of a manual tactile test for predicting precision pinch performance and disease severity in subjects with carpal tunnel syndrome. Arch Phys Med Rehabil 2014;95:717-25.

45. Jollis JG, Ancukiewicz M, DeLong ER, et al. Discordance of databases designed for claims payment versus clinical information systems. Implications for outcomes research. Ann Intern Med 1993; 119:844-50.

46. Remick RA. Diagnosis and management of depression in primary care: a clinical update and review. CMAJ 2002;167:1253-60. 